\title{
MULTICOLOR CCD PHOTOMETRY OF A HOMOGENEOUS SAMPLE OF SEYFERT 1 GALAXIES
}

\author{
C.BONOLI ${ }^{1}$, F.BONOLI ${ }^{2}$, L.DANESE ${ }^{3}$, F.DELPINO ${ }^{4}$ \\ G.DE ZOTTI ${ }^{1}$, G.GRANATO ${ }^{3}$, V.ZITELLI ${ }^{2}$ \\ 1 Osservatorio Astronomico, vicolo Osservatorio 5, 35122 - Padova, ITALY \\ 2 Dipartimento di Astronomia, via Zamboni 33, 40126 - Bologna, ITALY \\ 3 Dipartimento di Astronomia, vicolo Osservatorio 5, 35122 - Padova, ITALY \\ 4 Osservatorio Astronomico, via Zamboni 33, 40126 - Bologna, ITALY
}

ABSTRACT. We have observed a homogeneous sample of Seyfert 1 and 1.5 galaxies with the aim to determine the luminosity function of Seyfert nuclei. Observations were made with the CCD cameras of Asiago and Loiano Observatories in B, V and R bands in order to isolate the contribution of different stellar populations. A photometric decomposition into three components (disc, bulge and nucleus) was fitted to the observed luminosity profiles to separate the nuclear and the host galaxy contributions and to understand whether underlying galaxies actually behave as "normal" galaxies.

The properties of active galaxies have so far been studied mainly by means of spectroscopic investigations of the nuclei. However, a complete photometric study of the whole galaxy would be an important step toward the understanding of their behaviour.

The main goals of our study are the following. First, the determination of the luminosity function of Seyfert nuclei. Second, by determining properties of host galaxies, such as color, size and luminosity, to infer which kind of galaxies preferably contain active nuclei. Third, the comparison of galaxian properties with fuzziness around quasars.

The selected sample is described in Cheng et al. (1985) and is mainly extracted from the first nine lists of Markarian. It consists of 57 Seyfert 1 and 1.5 galaxies and is a homogeneous sample in the sense that it is possible to derive a meaningful luminosity function and incorporates corrections for incompleteness and for random errors.

A parallel project for the same galaxy sample is carried out in the near-infrared (Zitelli et al., 1988); moreover, the availability of observations from $X$ band to radio band for a large fraction of galaxies in the sample, together with our optical observations, will make possible a detailed multi-frequency analysis of the sample and will allow us to understand the connections between the different emission components.

Observations were carried out with the CCD cameras of Asiago and Loiano Observatories; systems are described in detail in Bortoletto and D'Alessandro (1985) and Bregoli et al. (1986), respectively. Johnson and Kron-Cousins systems of $B, V$ and $R$ filters were used.

After a first standard pre-reduction (dark subtraction, flat field correction, etc.), the data reduction proceeded in three steps. First, we sampled the background in section of the astronomical frames far from the objects and free of contamination of stars for the sky determination and subtraction. That is always a delicate point in doing surface photometry, especially when CCDs are used, owing to their small field of view.

In the second step, first one determines the center of the galaxy; the centering is done through the computation of the first momentum of the luminosity distribution around the lighest 
pixel. Then surface profiles were obtained by differentiating the integrated magnitudes measured in a sequence of 1-pixel-thick concentric circular apertures, going radially outward.In order to derive surface luminosity profiles, local intensity is sampled along each contour. The sampling points in general will not correspond to pixel centers, and some interpolation is necessary. That is a crucial point near the galaxy center, where intensity changes drastically and the contours are made of few points.

The aim of the third point is to isolate the contribution of the nuclear component from that of the underlying galaxy. To do so, we modeled surface profiles as the sum of three components: a point-like nucleus, an exponential disc and an $r^{1 / 4}$ bulge.

The observed profiles of each component then result from the convolution of the theoretical profile with a suitable point spread function. An estimate of the point spread function was obtained for each frame fitting the sum of two Gaussian curves to the stellar profiles.

The model depends on a set of parameters whose values are determined either on the frames (point spread function, b/a ratio, etc.) or by the comparison of the theoretical profile with the observed one (central intensity of the luminosity, scale factors, etc). For a given set of parameters, the synthetic profile of the galaxy is compared with the observed one, then minimizing the residuals in order to find the best values for the parameters of the fit. Figure 1 gives an example of the fitting of the profiles.

The reduction of the images is still in progress. A first conclusion we can draw from our data is that bulge is important in almost all processed profiles; that confirms, from the photometrical point of view, the finding of Heckman (1978), who analysed the morphology of active galaxies and found that they preferably are early-type spiral galaxies.

\section{References}

Bortoletto F. \& D'Alessandro M. : Rev. Sci. Instruments, 57, 253 (1986)

Bregoli G., Federici L., Focardi P., Merighi R., Oculi L., Piccioni A., Volta O. \& Zitelli V.: In The Optimization of the Use of CCD Detectors in Astronomy, Ed. J.P. Baluteau \& S. D'Odorico, 177 (1986)

Cheng Fu-zhen, Danese L., De Zotti G. \& Franceschini A. : Mon. Not. Roy. Ast. Soc., 212, $857(1985)$

Heckman T. : Pub. A.S.P., $\underline{90}, 241$ (1978)

Zitelli V., Wade R., Danese L., De Zotti G. \& Mandolesi N. : Present Symposium

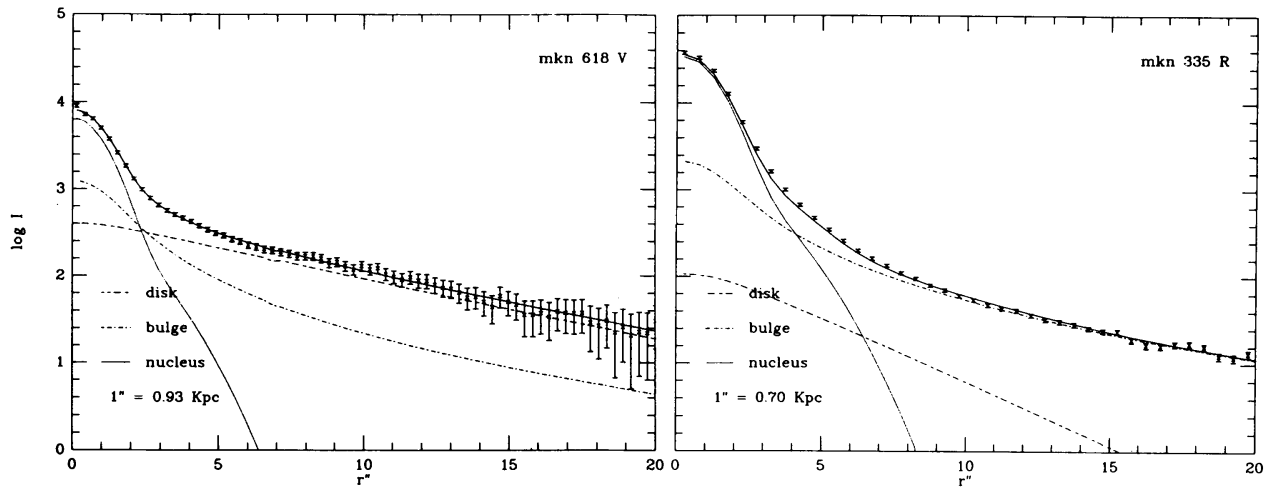

Figure 1. Profile decomposition. 